\title{
The Effects of Pressure and Temperature on the Magnetic Susceptibility of Semiconductor Quantum Dot in A Magnetic Field
}

\author{
Faten BZOUR ${ }^{1}$, Mohammad K. ELSAID ${ }^{1} \&$ Ayham SHAER ${ }^{1}$ \\ ${ }^{1}$ Physics Department, Faculty of Science, An- Najah National University, Nablus, West Bank, Palestine \\ Correspondence: Mohammad K. ELSAID, Physics Department, Faculty of Science, An- Najah National \\ University, Nablus, West Bank, Palestine. E-mail: mkelsaid@najah.edu
}

Received: November 25, 2016

Accepted: December 5, 2016

Online Published: January 2, 2017

doi:10.5539/apr.v9n1p77

URL: http://dx.doi.org/10.5539/apr.v9n1p77

\begin{abstract}
In this work, we present a theoretical study of the magnetic susceptibility $(\chi)$ of two-electron GaAs parabolic quantum dot (QD) under the combined effects of external pressure, temperature and magnetic field. We used the exact diagonalization method to obtain the eigenenergies by solving the two electron quantum dot Hamiltonian taking into account the dependence of the effective mass and dielectric constant on the hydrostatic pressure and temperature. The pressure and temperature show significant effects on the calculated QD spectra. Next, we investigate the behavior of the magnetization of a quantum dot as a function of external pressure, temperature, confining frequency and magnetic field. The singlet-triplet transitions in the ground state of the quantum dot spectra and the corresponding jumps in the magnetic susceptibility spectra have been shown. The comparison shows that our results are in very good agreement with the reported works.
\end{abstract}

Keywords: Pressure; temperature; magnetic susceptibility; quantum dot, magnetic field; exact diagonalization

\section{Introduction}

Recent nanofabrication methods have it possible to design different types of quantum dots with the flexibility of controlling the size, shape, and number of electrons. These controllable physical properties of the zero-dimensional nanostructure makes it promising candidate for a wide range of device applications like quantum dot lasers, solar cells, single electron transistors and quantum computers (Ashoori et al., 1993; Ciftja, 2013; Kastner, 1992; Loss \& DiVincenzo, 1998; Burkard, Loss, \& DiVincenzo, 1999).

Different approaches had been used to solve the two interacting electrons QD- Hamiltonian, including the presence of an applied magnetic field, and had obtained the eigenenergies and eigenstates of the QD-system as a function of magnetic field strength (Wagner, Merkt, \& Chaplik, 1992; Taut, 1994; Ciftja \& Kumar, 2004; Kouwenhoven, Austing, \& Tarucha, 2001; Sanjeev Kumar, Mukhopadhyay, \& Chatterjee, 2016; Kandemir, 2005; El-Said, 1995; El-Said, 1998; El-Said, 2000; Elsaid, Al-Naafa, \& Zugail, 2008; Maksym \& Chakraborty, 1990; De $\quad$ Groote, Hornos, \& Chaplik, 1992). The energy spectra shows spin-singlet (S) and spin-triplet (T) ground state oscillations. These spin oscillations show themselves as transition peaks in the spectra of magnetic and thermodynamic quantities like magnetization $(M)$, magnetic susceptibility $(\boldsymbol{\chi})$ and heat capacity $\left(C_{v}\right)$ (Nguyen \& Peeters, 2008; Nammas, Sandouqa, Ghassib, \& Al-Sugheir, 2011; Boyacioglu \& Chatterjee, 2012; Helle, Harju, \& Nieminen, 2005; Schwarz et al., 2002; Räsänen et al., 2003; Climente, Planelles, \& Movilla, 2004; Nguyen \& Sarma, 2011; Rezaei \& Kish, 2012; Dybalski \& Hawrylak, 2005; Avetisyan, Chakraborty, \& Pietiläinen, 2016).

The aim of this work, is to investigate the magnetic susceptibility of two interacting electrons confined in a parabolic quantum dot which is presented in a magnetic field. The applied magnetic field is uniform and its direction is taken to be along z-axis that is perpendicular to the $x-y$ plane of the QD. In addition, we consider the effects of the external pressure and temperature on the magnetic susceptibility curve. We initially applied the exact diagonalization method to solve the QD Hamiltonian and obtain the eigenenergies for various values of physical QD parameters. Secondly, we investigate the dependence of the QD magnetic susceptibility, as a thermodynamic quantity, on the pressure, temperature confining frequency and magnetic field strength.

The rest of this paper is organized as follows: section II presents the Hamiltonian theory, computation diagonalization technique and the statistical thermodynamic relations of magnetic susceptibility for two 
interacting electrons in the quantum dot. In section III, we give the numerical results for the energy spectra and the magnetic susceptibility of the QD. We devoted the final section for conclusions.

\section{Theory}

In this section we describe in detail the main two parts of the theory, namely: quantum dot Hamiltonian and exact diagonalization method and the magnetic susceptibility of GaAs quantum dot.

\subsection{Quantum Dot Hamiltonian}

The effective mass Hamiltonian for two interacting electrons confined in a QD by a parabolic potential in a uniform magnetic field $\vec{B}=B \hat{k}$ can be written in a separable form as:

$$
\begin{gathered}
\widehat{H}=\widehat{H}_{\mathrm{CM}}+\widehat{H}_{\mathrm{r}} \\
\widehat{H}_{\mathrm{CM}}=\frac{1}{2 \mathrm{M}}\left[\overrightarrow{\mathrm{P}}_{\mathrm{R}}+\frac{\mathrm{Q}}{\mathrm{c}} \overrightarrow{\mathrm{A}}(\overrightarrow{\mathrm{R}})\right]^{2}+\frac{1}{2} \mathrm{M} \omega_{0}^{2} \mathrm{R}^{2} \\
\widehat{H}_{\mathrm{r}}=\frac{1}{2 \mu}\left[\overrightarrow{\mathrm{p}}_{\mathrm{r}}+\frac{\mathrm{q}}{\mathrm{c}} \overrightarrow{\mathrm{A}}(\overrightarrow{\mathrm{r}})\right]^{2}+\frac{1}{2} \mu \omega_{0}^{2} \mathrm{r}^{2}+\frac{\mathrm{e}^{2}}{\epsilon|\overrightarrow{\mathrm{r}}|}
\end{gathered}
$$

Where $\omega_{0}$ is the confining frequency, $\mu=\frac{m^{*}}{2}$ is the reduced mass, $\mathrm{M}=2 \mathrm{~m}$ is the total mass, $q=\frac{e}{2}$ is the reduced charge, $\mathrm{Q}=2 \mathrm{e}$ is the total charged and $\epsilon$ is the dielectric constant for the GaAs medium. $\vec{R}=$ $\frac{\overrightarrow{r_{1}}+\overrightarrow{r_{2}}}{2}$ and $\vec{r}=\overrightarrow{r_{2}}-\overrightarrow{r_{1}}$ are the center of mass and relative coordinates, respectively. $\omega_{\mathrm{c}}=\frac{e B}{m^{*}}$ is the cyclotron frequency and $\mathbf{A}=\frac{1}{2} \mathbf{B} \times \mathbf{r}$ is the vector potential.

The corresponding energy of this Hamiltonian equation (1) is:

$$
E_{\text {total }}=E_{C M}+E_{r}
$$

The center of mass Hamiltonian given by equation (2) is a harmonic oscillator type with well-known eigenenergies:

$$
E_{c m}=\mathrm{E}_{\mathrm{n}}, \mathrm{m}_{\mathrm{cm}}=\left(2 \mathrm{n}_{\mathrm{cm}}+\left|\mathrm{m}_{\mathrm{cm}}\right|+1\right) \hbar \omega+\mathrm{m}_{\mathrm{cm}} \frac{\hbar \omega_{\mathrm{c}}}{2}
$$

where $\mathrm{n}_{\mathrm{cm}}, \mathrm{m}_{\mathrm{cm}}$ and $\omega=\sqrt{\frac{\omega_{c}^{2}}{4}+\omega_{0}^{2}}$ are the radial, angular quantum numbers and effective confining frequency, respectively.

However, the relative motion Hamiltonian part $\left(\mathrm{H}_{\mathrm{r}}\right)$, given by equation (3) does not have an analytical solution for all ranges of $\omega_{0}$ and $\omega_{c}$. In this work, we applied the exact diagonalization method to solve the relative part of the Hamiltonian and obtain the corresponding eigenenergies $E_{r}$.

\subsection{Exact Diagonalization Method and Magnetic Susceptibility}

For non-interacting case the relative Hamiltonian in equation (3) is a single particle problem with eigenstates $\mid n_{r} m_{r}>$ (Kouwenhoven, Austing, \& Tarucha, 2001),

$$
\left|\mathrm{n}_{\mathrm{r}} \mathrm{m}_{\mathrm{r}}\right\rangle=\mathrm{N}_{n_{r} \mathrm{~m}_{\mathrm{r}}} \frac{\mathrm{e}^{\mathrm{im} \mathrm{r} \phi}}{\sqrt{2 \pi}}(\alpha \mathrm{r})^{\left|\mathrm{m}_{\mathrm{r}}\right|} \mathrm{e}^{-\alpha^{2} \mathrm{r}^{2} / 2} \mathrm{~L}_{\mathrm{n}_{\mathrm{r}}}^{\left|\mathrm{m}_{\mathrm{r}}\right|}\left(\alpha^{2} \mathrm{r}^{2}\right)
$$

where the functions $L_{n_{r}}^{\left|m_{r}\right|}\left(\alpha^{2} r^{2}\right)$ are the standard associated Laguerre polynomials. We calculated the normalization constant $\mathrm{N}_{n_{r} \mathrm{~m}_{\mathrm{r}}}$ from the normalization condition of the basis, $<n_{r} m_{r} \mid n_{r} m_{r}>=1$, which resulted in

$$
N_{n_{r m_{r}}}=\sqrt{\frac{2 \mathrm{n}_{\mathrm{r}} ! \alpha^{2}}{\left(\mathrm{n}_{\mathrm{r}}+\mid \mathrm{m}_{\mathrm{r}}\right) !}}
$$

We used $\alpha$ as a constant which has the dimensionality of an inverse length 


$$
\alpha=\sqrt{\frac{\mathrm{m} \omega}{\mathrm{h}}}
$$

The eigenenergies of the QD Hamiltonian which are given by equation (4) consist of the sum of the energies for the center of mass Hamiltonian $\left(E_{c m}\right)$ and the eigenenergies $\left(E_{r}\right)$ which are obtained by direct diagonalization to the relative Hamiltonian part. For interacting case, we applied the diagonalization method to solve equation (3) and find the corresponding exact eigenenergies for arbitrary values of $\omega_{c}$ and $\omega_{0}$.

We can write the matrix element of the relative Hamiltonian part using the basis $\mid n_{r} m_{r}>$ as,

$$
\begin{aligned}
\mathrm{h}_{\mathrm{nn}^{\prime}}=\left\langle\mathrm{n}_{\mathrm{r}}, \mathrm{m}_{\mathrm{r}}\left|\widehat{\mathrm{H}}_{\mathrm{r}}\right| \mathrm{n}^{\prime}{ }_{\mathrm{r}}, m_{\mathrm{r}}\right\rangle & \left.=<n_{r} m_{r}\left|-\frac{\hbar^{2}}{2 \mu} \nabla^{2}+\frac{1}{2} \mu \omega^{2} r^{2}\right| n_{r}^{\prime} m_{r}\right\rangle+ \\
& \left.<n_{r} m_{r}\left|\frac{e^{2}}{\epsilon \vec{r}}\right| n_{r}^{\prime} m_{r}\right\rangle
\end{aligned}
$$

The first term in the right side of equation (9) is diagonalized as,

$$
\left[\left(2 \mathrm{n}+\left|m_{z}\right|+1\right) \sqrt{\left(1+\frac{\gamma^{2}}{4}\right)}-\frac{\gamma}{2}\left|m_{z}\right|\right] \delta_{n n^{\prime}}
$$

Where the coulomb matrix energy can be given as

$$
\frac{\lambda}{\sqrt{2}} \sqrt{\frac{\mathrm{n}^{\prime} ! \mathrm{n} !}{\left(\mathrm{n}^{\prime}+\left|m_{z}\right|\right) !\left(\mathrm{n}+\left|\mathrm{m}_{\mathrm{z}}\right|\right) !}} \times \mathrm{I}_{\mathrm{nn}^{\prime}}\left|m_{z}\right|
$$

where $\gamma=\frac{\omega_{c}}{\omega_{0}}$ and $\lambda=\frac{\mathbf{e}^{2} \boldsymbol{\alpha}}{\hbar \omega}$ are dimensionless parameters while $\omega^{2}=1+\frac{\gamma^{2}}{4}$ is the effective confining frequency.

By changing the coordinate transformation to t-variable by direct substitution of $r=\frac{\sqrt{t}}{\alpha}$ in the integrationI $\mathrm{nn}^{\prime}=$ $I_{n_{r} n_{r}^{\prime}}$, we can express the coulomb energy matrix element into the integral form:

$$
<n_{r} m_{r}\left|\frac{e^{2}}{\epsilon r}\right| n_{r}^{\prime} m_{r}>\propto I_{\mathrm{nn}^{\prime}\left|\mathrm{m}_{\mathrm{z}}\right|}=\int_{0}^{\infty} \mathrm{dt} \mathrm{t}^{\left|\mathrm{m}_{\mathrm{z}}\right|} \mathrm{e}^{-\mathrm{t}} \mathrm{L}_{\mathrm{n}}^{\left|\mathrm{m}_{\mathrm{z}}\right|}(\mathrm{t}) \mathrm{L}_{\mathrm{n}^{\prime}}^{\left|\mathrm{m}_{\mathrm{z}}\right|}(\mathrm{t}) \frac{1}{\sqrt{\mathrm{t}}}
$$

We evaluated the above coulomb energy matrix element in a closed form by using the Laguerre relation given in the appendix A (Nguyen \& Sarma, 2011).

This closed form result of the coulomb energy reduces greatly the computation time needed in the diagonalization process.

In our calculation, we have used the basis $\mid n_{r} m_{r}>$ defined by equation (6) to diagonalize the relative QD Hamiltonian and obtain its corresponding eigenenergies $E_{r}$. The exact diagonalization method is used in spanning the total Hamiltonian for the selected single electron basis and extract the lowest eigenvalues (eigenenergies) of the matrix. The procedure of increasing the number of linearly independent eigenstates is converging to the exact results. In each step the new energy results are compared with previous results from a smaller apace, until satisfactory convergence is achieved.

Next step, we have calculated the magnetic susceptibility $(\boldsymbol{\chi})$ from the mean energy $\langle E(T)\rangle$ and the magnetization $(\mathrm{M})$ of the two-electron quantum dot using the statistical thermodynamic relations:

$$
M=\frac{\partial\langle E(T)\rangle}{\partial B} \quad \text { and } \quad \chi=\frac{\partial M}{\partial B},
$$

where, the statistical energy $\langle E(T)\rangle$ is obtained by the help of the partition function.

To include the effect of the pressure $(\mathrm{P})$ and temperature $(\mathrm{T})$ on the QD energy states and the magnetization we replace the dielectric constant $\epsilon$ with $\epsilon_{r}(P, T)$ and the effective mass $m^{*}$ with $m(P, T)$ in the QD Hamiltonian as defined by Equations 2 and 3, where $\epsilon_{r}(P, T)$ and $m^{*}(P, T)$ are the pressure and temperature dependent dielectric constant and electron effective mass, respectively. These pressure and temperature dependent mass parameters should be included in the energy spectrum Eq.4 and the wave functions basis eq. 6 of the Hamiltonian. For quantum dot made of GaAs the dependency of $\epsilon_{r}(P, T)$ and $m^{*}(P, T)$ are given in appendix B (Rezaei \& Kish, 2012). 
The pressure and temperature effective Rydberg $\left(R_{y}^{*}(P, T)\right)$ is used as the energy unit and given as follows:

$$
R_{y}^{*}(P, T)=\frac{e^{2}}{2 \epsilon(P, T) a_{B}^{*}(P, T)}
$$

Where $a_{B}^{*}(P, T)$ is the effective Bohr radius and given as:

$$
a_{B}^{*}(P, T)=\epsilon(P, T) \hbar^{2} /\left(m^{*}(P, T) e^{2}\right)
$$

So the effective Rydberg can be written as:

$$
R_{y}^{*}(P, T)=\frac{e^{4} m^{*}(P, T)}{2(\epsilon(P, T))^{2} \hbar^{2}}
$$

We change the pressure and temperature values to see their effects on the ground state energy of the QD Hamiltonian in both cases: zero $\left(\omega_{c}=0\right)$ and finite magnetic field $\left(\omega_{c}\right)$. Eventually, the ground state energies of the two electron-quantum dot system will be calculated as function of temperature $(\mathrm{T})$, pressure $(\mathrm{P})$, confining frequency $\left(\omega_{0}\right)$ and magnetic field cyclotron frequency $\left(\omega_{c}\right)$.The obtained numerical results are displayed in the next section.

\section{Results and Discussions}

We present the effects of pressure, temperature, confining frequency and magnetic field cyclotron frequency on the magnetic susceptibility of two interacting electrons in a quantum dot made from GaAs material (effective Rydberg $\mathrm{R}^{*}=5.825 \mathrm{meV}$ ) in Figures 1 to 5 and Table 1 . To achieve our aim, it is essential, as a first step, to investigate the dependence of the QD energy levels on the pressure and temperature. In Figure 1, we display the dependence of the QD energy states $(\mathrm{m}=0,1,2,3$ and 4$)$ on the magnetic field, $\omega_{c}$, for pressure $\mathrm{P}=10 \mathrm{Kbar}$ and temperature $\mathrm{T}=0.0 \mathrm{~K}$. We found that the overall shape of the spectra of the QD remains the same while the eigenenergies are enhanced under the effect of external pressure. For zero magnetic field and zero pressure case, we have tested in Table 1, the computed numerical results against the corresponding ones taken from the work of Ciftja and Kumar (Ciftja \& Kumar, 2004). The comparisons give excellent agreement between the energy spectra of two-electron QD Hamiltonian solved by both exact numerical and variational methods (Ciftja \& Kumar, 2004; Ciftja \& Kumar, 2004; Kandemir, 2005; Dybalski \& Hawrylak, 2005). The QD spectra shows transitions in the ground state angular momentum $(\mathrm{m})$ as the magnetic field increases. For example, we observed the first transitions in the angular momentum of the ground state of the QD system, from $\mathrm{m}=0$ to $\mathrm{m}=1$, occurs at $\omega_{c} \approx 0.8 R^{*}$ while the second transition ( from $\mathrm{m}=1$ to $\mathrm{m}=2$ ) occurs at $\omega_{c} \approx 1.2 R^{*}$. These transitions show themselves as cusps in the presented QD-magnetization curves. Figure 2, displays the energies of the quantum dot state $(\mathrm{m}=0)$ against the magnetic field for various pressure values: $\mathrm{P}=0,10,20$ and $30 \mathrm{Kbar}$ and Temperature $\mathrm{T}=0.0$. For fixed particular value of the magnetic field, the figure clearly shows an enhancement in the energy level as the pressure increases. As the pressure increases the dielectric constant decreases leading to a significant electron-electron coulomb energy enhancement. Next we show the dependence of the magnetic susceptibility of the QD on the pressure, temperature and magnetic field. Figures $3 a$ and $3 b$ show the effects of the pressure on the dependence of the magnetic susceptibility on magnetic field. The magnetic susceptibility plotting is given for no external pressure ( $\mathrm{P}=0.0$ ) and for pressure value, $\mathrm{P}=30.0 \mathrm{Kbar}$, at temperature $\mathrm{T}=.01 \mathrm{~K}$ and confining energy frequency $\omega_{0}=$ $0.5 R^{*}$. Figures $3 \mathrm{a}$ and $3 \mathrm{~b}$ show that as the pressure increases: $\mathrm{P}=0.0 \mathrm{Kbar}$ to $30 \mathrm{Kbar}$, the absolute value of the magnetic susceptibility $(|\boldsymbol{X}|)$ at $\omega_{c}=0.0$ also increases. In addition the heights of the peaks enhance as the magnetic field increases .For example, we can observe the changes in the height of peak number five which is located at $\omega_{c} \approx 3.8 R^{*}$. This magnetic susceptibility behavior, under the effect of pressure, can be attributed to the enhancement of the electron-electron repulsive coulomb interaction energy in the quantum dot. As the pressure increases the dielectric constant, $\epsilon(p)$, decreases and thus the coulomb interaction energy term, $v_{c} \approx \frac{1}{c(p) r}$, in the QD-Hamiltonian, enhances. This leads to a significant energy increase in the total energy state of the electron making the electron unstable. The electron in this case makes a jump to another state with higher angular momentum ( $\mathrm{m}$ ) in order to reduce its coulomb energy, and in effective its total energy decreases, to become more stable. Figure 4 shows the effect of temperature on the QD magnetic susceptibility plot by changing the temperature from $\mathrm{T}=0.01 \mathrm{~K}$ (Figure $3 \mathrm{a}$ ) to $\mathrm{T}=1 \mathrm{~K}$ (Figure 4 ) for no external pressure $(\mathrm{P}=0.0 \mathrm{Kbar}$ ) and the same confining frequency, $\omega_{c}=0.5 R^{*}$. The magnetic susceptibility plot shows again a significant temperature dependence. We can clearly see the significant reduction in the height of magnetic susceptibility peaks as the temperature increases from $\mathrm{T}=0.01 \mathrm{~K}$ to $\mathrm{T}=1 \mathrm{~K}$.For example, only the first peak located at $\omega_{c}=0.4 R^{*}$ remains, while the rest of the peaks at high magnetic field strength almost disappear due to the thermal fluctuations and the magnetic susceptibility becomes smooth. Furthermore, we study, in Figure 5, the effect of confining frequency $\omega_{0}$ on the shape of the magnetic susceptibility spectra. We consider different values of confining frequencies: 
$\omega_{0}=0.5$ and $0.8 R^{*}$ keeping the temperature and the pressure parameters both are unchanged: $\mathrm{P}=0.0 \mathrm{kbar}$ and $\mathrm{T}=0.01 \mathrm{~K}$. The magnetic susceptibility spectra calculated at $\omega_{0}=0.5 R^{*}$ and $\omega_{0}=0.8 R^{*}$ are given in Figure $3 \mathrm{a}$ and Figure 5, respectively. The comparison of the spectra in both figures clearly shows a significant confining frequency dependence $\omega_{0}$, which results in shifting the peak positions of the magnetic susceptibility spectra as clearly shown. These peaks- shift behavior can be understood in this way. As we increase the confining frequency, $\omega_{0}$, the electron in the quantum dot becomes more confined and this electron energy enhancement shifts significantly the location of the energy level crossings to a higher magnetic field strength. For example, our confining frequencies: $\omega_{0}=0.5$ and $0.8 R^{*}$ corresponds to transition magnetic field values: $\omega_{c}=$ 0.4 and $0.9 R^{*}$, respectively. The eigenenergies are obtained by diagonalizing the full QD-Hamiltonian matrix given by equation (9). In all steps of calculations, the numerical convergence is achieved. For example, the ground state energy calculated at $\lambda=3$ in Table 1, converges to $E_{r}=4.324,4.320,4.319$ and $4.319 \mathrm{meV}$ as the number of basis in the matrix elements $S_{p}=5,20,40$ and 50, respectively.

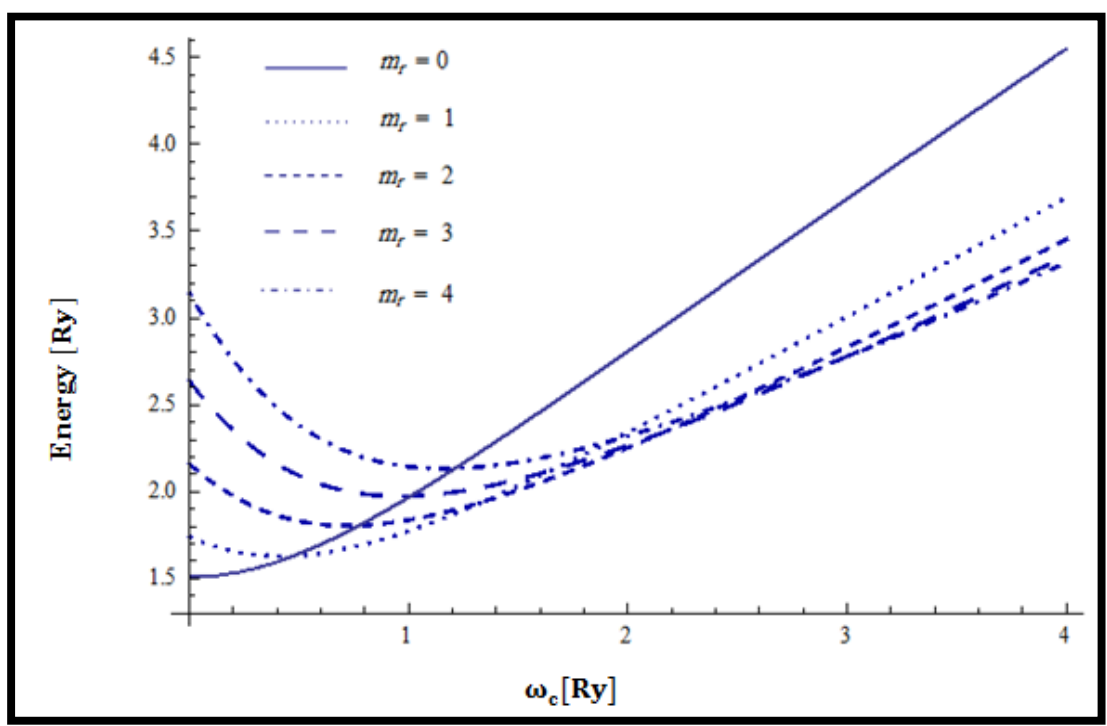

Figure 1. The computed energy spectra of quantum dot versus the strength of the magnetic field for $\omega_{0}=0.5 \mathrm{R}^{*}$, $\mathrm{T}=0 \mathrm{~K}, \mathrm{P}=10 \mathrm{Kbar}$ and angular momentum $\mathrm{m}=0,1,2,3,4$

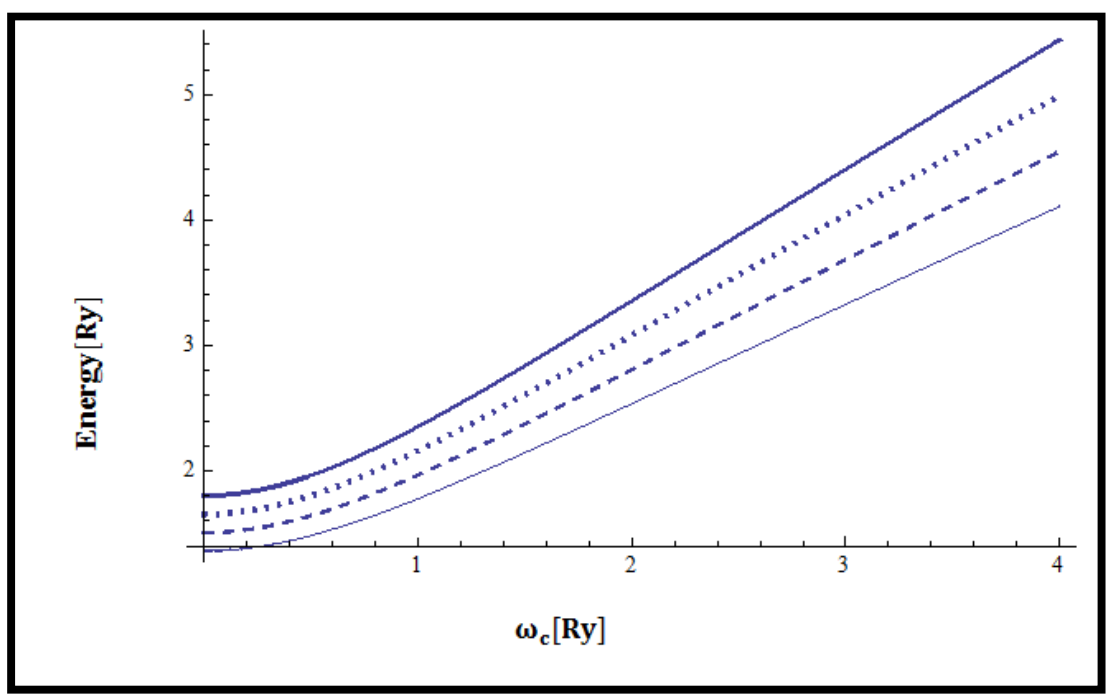

Figure 2. The computed energy spectra of quantum dot versus the strength of the magnetic field for $\boldsymbol{\omega}_{\mathbf{0}}=\mathbf{0} . \mathbf{5} \mathbf{R}^{*}$, $\mathrm{T}=0 \mathrm{~K}, \mathrm{~m}=0$ and various pressures $(\mathrm{P}=0 \mathrm{Kbar}$ solid; $\mathrm{P}=10 \mathrm{Kbar}$ dashed; $\mathrm{P}=20 \mathrm{Kbar}$ dotted and $\mathrm{P}=30 \mathrm{Kbar}$ thick $)$ 


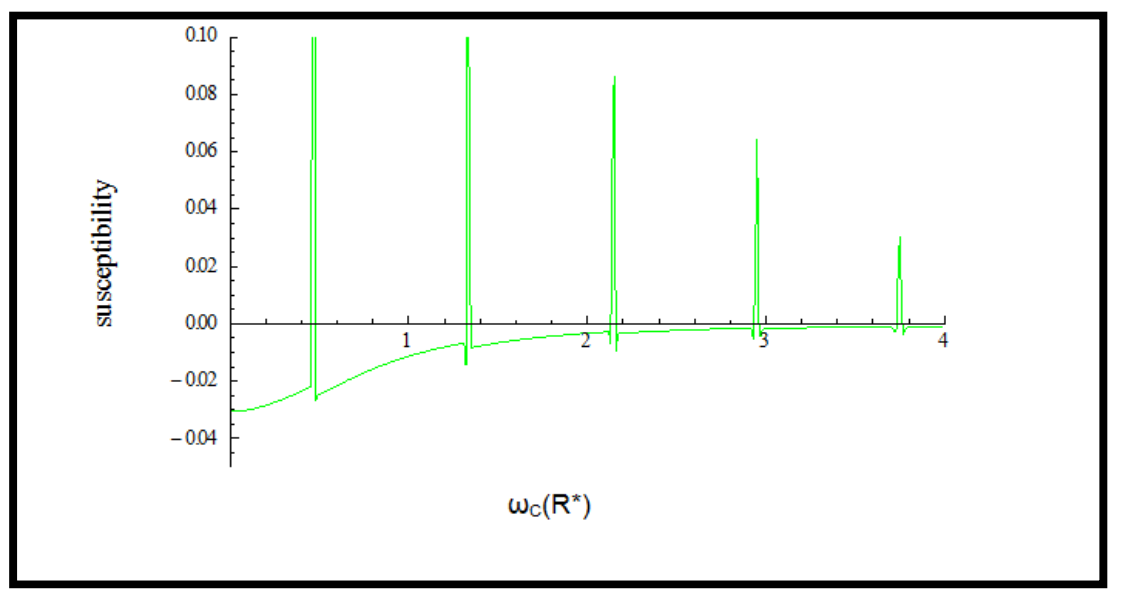

Figure 3a

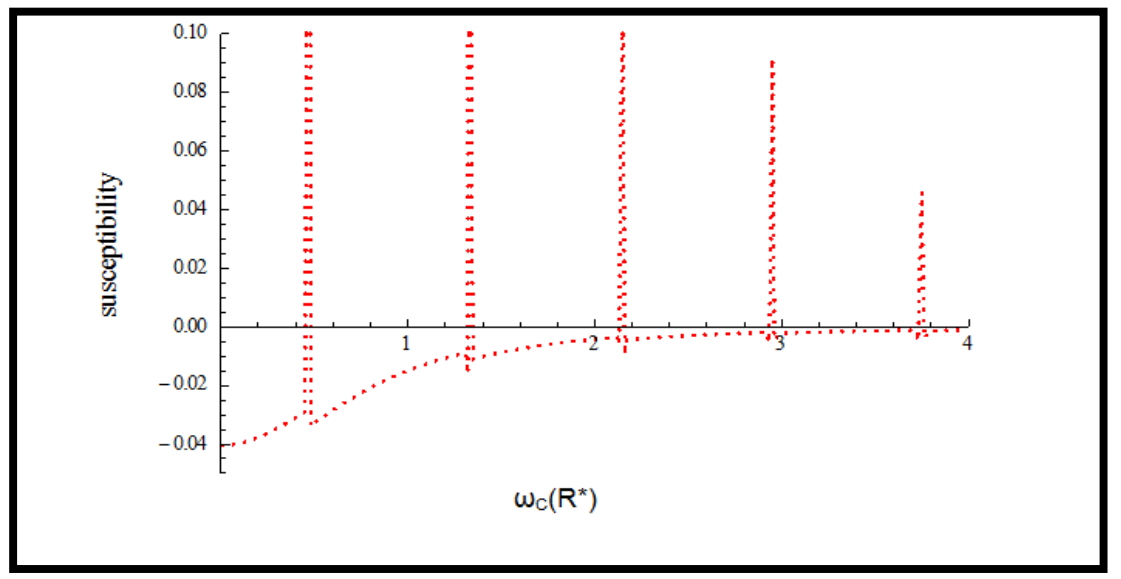

Figure 3b

Figure 3. Magnetic susceptibility ( $\chi$ ) of the two electrons quantum dot as function of magnetic field strength, calculated at fixed confining frequency $\boldsymbol{\omega}_{\mathbf{0}}=\mathbf{0} . \mathbf{5} \boldsymbol{R}^{*}$, pressure $\mathrm{T}=0.01 \mathrm{~K}$ and different temperatures : a) $\mathrm{P}=0.0$ Kbar and b) $\mathrm{P}=3.0 \mathrm{Kbar}$

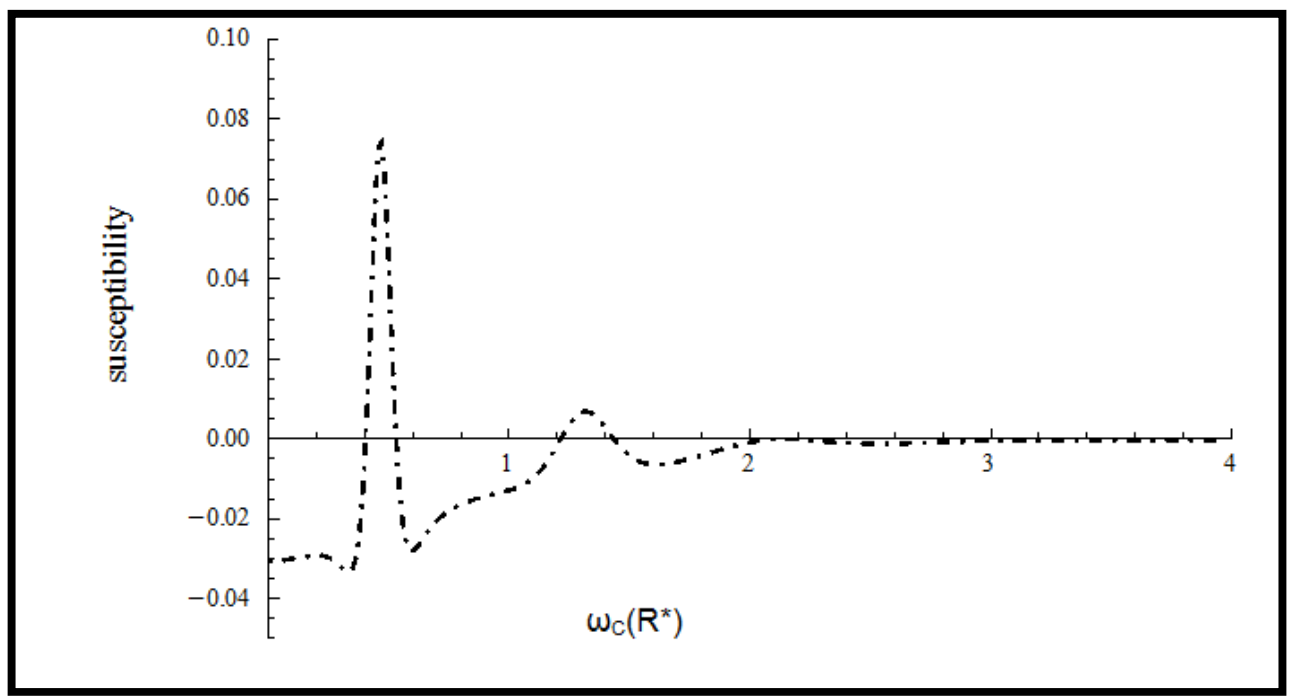

Figure 4. Magnetic susceptibility ( $\chi$ ) of the quantum dot as function of magnetic field strength at fixed confining frequency $\omega_{0}=0.5 R^{*}$, Temperature $\mathrm{T}=1 \mathrm{~K}$ and pressure $\mathrm{P}=0 \mathrm{Kbar}$ 


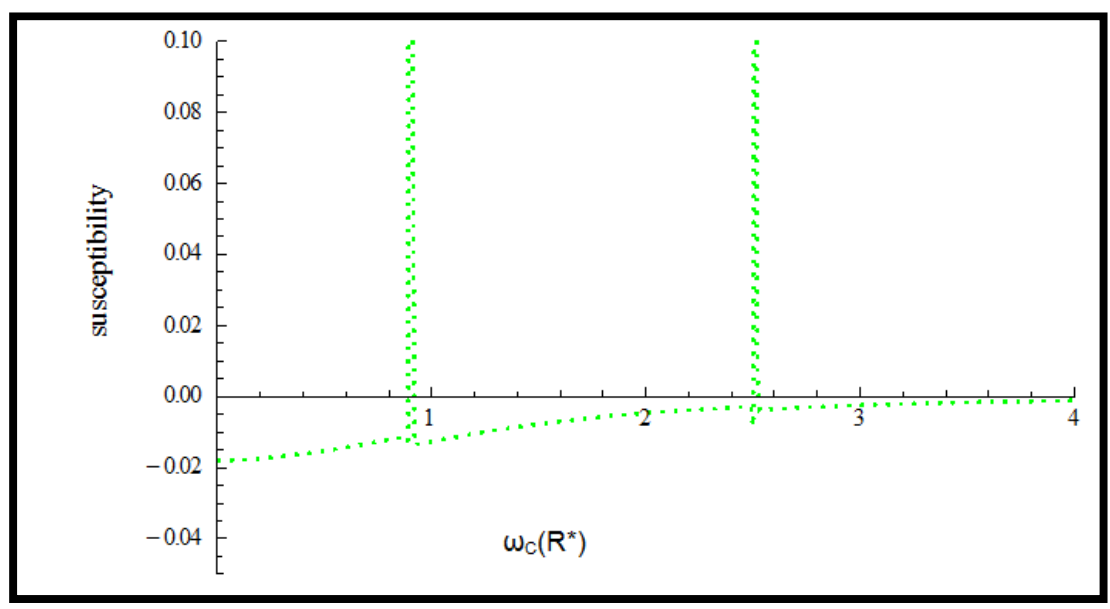

Figure 5. Magnetic susceptibility $(\chi)$ of the quantum dot as function of magnetic field strength at fixed confining frequency $\omega_{0}=0.8 R^{*}$, Temperature $\mathrm{T}=0.01 \mathrm{~K}$ and pressure $\mathrm{P}=0 \mathrm{Kbar}$

Table 1. The ground state energies of QD (in $R^{*}$ ) as a function of dimensionless coulomb coupling parameter $\lambda$ obtained from exact diagonalization method (second column) compared with reported work (third column) Reference (Ciftja \& Kumar, 2004)

\begin{tabular}{ccc}
\hline $\boldsymbol{\lambda}$ & E (Present work) & E(Ciftja \& Kumar, 2004) \\
\hline 0 & 2.00000 & 2.00000 \\
1 & 3.000969 & 3.00097 \\
2 & 3.721433 & 3.72143 \\
3 & 4.318718 & 4.31872 \\
4 & 4.847800 & 4.84780 \\
5 & 5.332238 & 5.33224 \\
6 & 5.784291 & 5.78429 \\
7 & 6.211285 & 6.21129 \\
8 & 6.618042 & 6.61804 \\
9 & 7.007949 & 7.00795 \\
10 & 7.383507 & 7.38351 \\
\hline
\end{tabular}

\section{Conclusions}

The electronic energy levels and the magnetic susceptibility of two interacting electrons in the quantum dot have been calculated as a function of pressure, temperature confining frequency and magnetic field. The magnetic susceptibility spectra shows some peak structures which correspond to the energy levels and the associated spin-singlet-triplet transitions in the ground state of the quantum dot. The calculations show that the absolute value of the magnetic susceptibility $(|\chi|)$ and the height of the transition peaks in the susceptibility spectra enhances also as the pressure increases, while keeping the values of temperature and magnetic field strength unchanged. In addition, the magnetic susceptibility spectra shows a temperature dependent behavior. The height and the number of the transition peaks in the magnetic susceptibility spectra changes significantly due to the thermal fluctuations. Furthermore, the confining frequency has a significant effect on the magnetic susceptibility spectra. We have observed that, as the confining frequency increases, the energy of the electrons increases and thus the positions of the energy level crossings are shifted towards a higher transition field strength. This in turn leads to changes in locations of the peaks spectra in the magnetic susceptibility spectra of the quantum dot.

\section{Appendix A: Properties of the Laguerre polynomials}

The following Laguerre relation was used to evaluate the coulomb energy matrix element in a closed form (Nguyen \& Sarma, 2011):

$$
\begin{gathered}
\int_{0}^{\infty} t^{\alpha-1} e^{-p t} L_{m}^{\lambda}(a t) L_{n}^{\beta}(b t) d t= \\
\frac{\Gamma(\alpha)(\lambda+1)_{m}(\beta+1)_{n} p^{-\alpha}}{m ! n !} \sum_{j=0}^{m} \frac{(-m)_{j}(\alpha)_{j}}{(\lambda+1)_{j} j !}\left(\frac{a}{p}\right)^{j} \sum_{k=0}^{n} \frac{(-n)_{k}(\alpha+j)_{k}}{(\beta+1)_{k} k !}\left(\frac{b}{p}\right)^{k}
\end{gathered}
$$


Appendix B: The pressure and temperature dependent dielectric constant and electron effective mass.

$$
\begin{gathered}
\in_{r}(P, T)=\left\{\begin{array}{c}
12.74 \exp \left(-1.73 \times 10^{-3} P\right) \exp \left[9.4 \times 10^{-5}(T-75.6)\right] \text { for } \mathrm{T}<200 \mathrm{~K} \\
13.18 \exp \left(-1.73 \times 10^{-3} P\right) \exp \left[20.4 \times 10^{-5}(T-300)\right] \text { forT } \geq 200 \mathrm{~K}
\end{array}\right. \\
m^{*}(P, T)=\left[1+7.51\left(\frac{2}{E_{g}^{\Gamma}(P, T)}+\frac{1}{E_{g}^{\Gamma}(P, T)+0.341}\right)\right]^{-1} m_{0} \\
E_{g}^{\mathrm{r}}(P, T)=\left[1.519-5.405 \times 10^{-4} \frac{T^{2}}{T+204}\right]+b P+c P^{2}
\end{gathered}
$$

Where $m_{0}$ is the free electron mass, $E_{g}^{\Gamma}(P, T)$ is the pressure and temperature dependent energy band gap for GaAs quantum dots at $\mathrm{r}$ point, $\mathrm{b}=1.26 \times 10^{-1} \mathrm{eV} \mathrm{GPa}^{-1}$ and $\mathrm{c}=-3.77 \times 10^{-3} \mathrm{eV} \mathrm{GPa}^{-2}$ (Rezaei \& Kish, 2012).

\section{References}

Ashoori, R. C., Stormer, H. L., Weiner, J. S., Pfeiffer, L. N., Baldwin, K. W., \& West, K. W. (1993). N-electron ground state energies of a quantum dot in magnetic field. Physical review letters, 71(4), 613.

Avetisyan, S., Chakraborty, T., \& Pietiläinen, P. (2016). Magnetization of interacting electrons in anisotropic quantum dots with Rashba spin-orbit interaction. Physica E: Low-dimensional Systems and Nanostructures, 81, 334-338.

Boyacioglu, B., \& Chatterjee, A. (2012). Heat capacity and entropy of a GaAs quantum dot with Gaussian confinement. Journal of applied physics, 112(8), 083514.

Burkard, G., Loss, D., \& DiVincenzo, D. P. (1999). Coupled quantum dots as quantum gates. Physical Review B, 59(3), 2070.

Ciftja, O. (2013). Understanding electronic systems in semiconductor quantum dots. Physica Scripta, 88(5), 058302.

Ciftja, O., \& Kumar, A. A. (2004). Ground state of two-dimensional quantum-dot helium in zero magnetic field: Perturbation, diagonalization, and variational theory. Physical Review B, 70(20), 205326.

Climente, J. I., Planelles, J., \& Movilla, J. L. (2004). Magnetization of nanoscopic quantum rings and dots. Physical Review B, 70(8), 081301.

De Groote, J. J. S., Hornos, J. E. M., \& Chaplik, A. V. (1992). Thermodynamic properties of quantum dots in a magnetic field. Physical Review B, 46(19), 12773.

Dybalski, W., \& Hawrylak, P. (2005). Two electrons in a strongly coupled double quantum dot: From an artificial helium atom to a hydrogen molecule. Physical Review B, 72(20), 205432.

El-Said, M. (1995). Two-electron quantum dots in a magnetic field. Semiconductor science and technology, 10(10), 1310.

El-Said, M. (1998). The energy level ordering in two-electron quantum dot spectra. Superlattices and microstructures, 23(6), 1237-1243.

El-Said, M. (2000). Spectroscopic structure of two interacting electrons in a quantum dot by the shifted $1 / \mathrm{N}$ expansion method. Physical Review B, 61(19), 13026.

Elsaid, M. K., Al-Naafa, M. A., \& Zugail, S. (2008). Spin Singlet-Triplet Energy Splitting in the Ground State of a Quantum Dot with a Magnetic Field: Effect of Dimensionality. Journal of Computational and Theoretical Nanoscience, 5(4), 677-680.

Helle, M., Harju, A., \& Nieminen, R. M. (2005). Two-electron lateral quantum-dot molecules in a magnetic field. Physical Review B, 72(20), 205329.

Kandemir, B. S. (2005). Variational study of two-electron quantum dots. Physical Review B, 72(16), 165350.

Kastner, M. A. (1992). The single-electron transistor. Reviews of Modern Physics, 64(3), 849.

Kouwenhoven, L. P., Austing, D. G., \& Tarucha, S. (2001). Few-electron quantum dots. Reports on Progress in Physics, 64(6), 701.

Loss, D., \& DiVincenzo, D. P. (1998). Quantum computation with quantum dots. Physical Review A, 57(1), 120.

Maksym, P. A., \& Chakraborty, T. (1990). Quantum dots in a magnetic field: Role of electron-electron interactions. Physical review letters, 65(1), 108. 
Nammas, F. S., Sandouqa, A. S., Ghassib, H. B., \& Al-Sugheir, M. K. (2011). Thermodynamic properties of two-dimensional few-electrons quantum dot using the static fluctuation approximation (SFA). Physica B: Condensed Matter, 406(24), 4671-4677.

Nguyen, N. T., \& Peeters, F. M. (2008). Magnetic field dependence of the many-electron states in a magnetic quantum dot: The ferromagnetic-antiferromagnetic transition. Physical Review B, 78(4), 045321.

Nguyen, N. T., \& Sarma, S. D. (2011). Impurity effects on semiconductor quantum bits in coupled quantum dots. Physical Review B, 83(23), 235322.

Räsänen, E., Saarikoski, H., Stavrou, V. N., Harju, A., Puska, M. J., \& Nieminen, R. M. (2003). Electronic structure of rectangular quantum dots. Physical Review B, 67(23), 235307.

Rezaei, G., \& Kish, S. S. (2012). Effects of external electric and magnetic fields, hydrostatic pressure and temperature on the binding energy of a hydrogenic impurity confined in a two-dimensional quantum dot. Physica E: Low-dimensional Systems and Nanostructures, 45, 56-60.

Sanjeev Kumar, D, Mukhopadhyay, S., \& Chatterjee, A. (2016). Magnetization and susceptibility of a parabolic InAs quantum dot with electron-electron and spin-orbit interactions in the presence of a magnetic field at finite temperature. Journal of Magnetisim and Magnetic Materials, 418, 169.

Schwarz, M. P., Grundler, D., Wilde, M., Heyn, C., \& Heitmann, D. (2002). Magnetization of semiconductor quantum dots. Journal of applied physics, 91(10), 6875-6877.

Taut, M. (1994). Two electrons in a homogeneous magnetic field: particular analytical solutions. Journal of Physics A: Mathematical and General, 27(3), 1045.

Wagner, M., Merkt, U., \& Chaplik, A. V. (1992). Spin-singlet-spin-triplet oscillations in quantum dots. Physical Review B, 45(4), 1951.

\section{Copyrights}

Copyright for this article is retained by the author(s), with first publication rights granted to the journal.

This is an open-access article distributed under the terms and conditions of the Creative Commons Attribution license (http://creativecommons.org/licenses/by/4.0/). 